\title{
Coarse-Grained Reconfigurable Stream Processor for Distributed Smart Cameras
}

\author{
Wei-Kai Chan, Yu-Hsiang Tseng, Yu-Sheng Lin, and Shao-Yi Chien \\ Media IC and System Lab \\ Graduate Institute of Electronics Engineering and Department of Electrical Engineering \\ National Taiwan University, Taipei 106, Taiwan \\ Email: sychien@ntu.edu.tw
}

\begin{abstract}
To support distributed video content analysis for video sensors in machine-to-machine networks, a reconfigurable stream processor for distributed smart cameras is proposed in this paper. A coarse-grained reconfigurable image stream processing architecture (CRISPA) with heterogeneous stream processing (HSP) and subword-level parallelism (SLP) is proposed to accelerate various algorithms for computer vision applications of smart-cameras. Implementation results show that the proposed design outperforms existing vision processors in many aspects: the on-chip memory size, power efficiency and area efficiency are 18.2 to 87.4 times, 4.5 to 12.5 times, and 3.8 to 25.5 times better than the state-of-the-art chips. Moreover, the programmability of the proposed design makes it capable of supporting many highlevel computer vision algorithms in high specification.
\end{abstract}

\section{INTRODUCTION}

Machine-to-machine (M2M) network, or Internet-ofThings (IoT), is thought as the next wave of information technology revolution [1], [2], where billions of ubiquitous intelligent devices, such as sensors, actuators, cellular phones, cars, and home appliances, are connected to communicate with each other under the information infrastructure composed of aggregators, gateways, and cloud servers. In M2M networks, sensors play important roles as physical data acquisition modules. Introducing visual information into M2M networks will lead to several new applications. However, the high power consumption, large transmission bandwidth, and high deployment cost of video sensors are the major design challenges. Thanks for the advances of semiconductor and computer vision technologies, distributed smart cameras [3]-[5], where video content analysis functions are integrated, are thought to be the key component to address these design challenges by converting raw image data into high-level context information at the front-end of the whole network, as shown in Fig. 1 [6]-[8]. To support the real-time processing and various functionalities in smart cameras, such as object segmentation/tracking, face detection, and object detection/recognition, the design of vision processors is required.

To design the vision processors for smart camera applications, there are many design challenges. First of all, high throughput, large memory size and high memory bandwidth are usually required. These requirements are the bottlenecks to be overcome for real-time applications of the computer vision algorithms. Second, in addition to real-time high performance, various smart camera applications, such as those mentioned above, should be considered at the same time. The requirement of programmability here makes ASIC approach

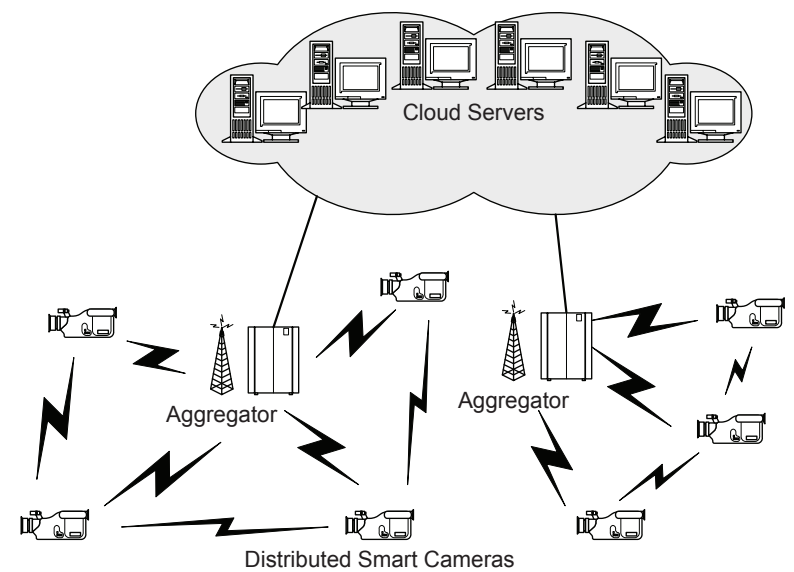

Fig. 1. Distributed smart camera as a node of an M2M network.

not appropriate. On the other hand, heterogeneous data types, such as 1-bit, 8-bit, 16-bit, 24-bit data, are usually required in the computer vision algorithm, which makes processorbased approaches inefficient. A processor architecture which can handle and balance the processing of different data types is required to enhance the processing throughput. In literatures, many vision processors and chips were proposed. However, most of them are designed with high hardware cost of Megabits on-chip memory and millions of gate counts [9]-[12]. Moreover, some of them only target at low frame resolutions, and the cost can be even higher or the throughput would be largely reduced for higher frame resolutions [10], [11].

In this work, a coarse-grained reconfigurable stream processor is proposed, which is characterized as follows. 1) The proposed coarse-grained reconfigurable image stream processing architecture (CRISPA) is employed to achieve high performance, programmability, low power and low cost. It is designed with coarse-grained reconfigurability, where processing units and memory elements are designed as the reconfigurable fabrics for programming and hardware sharing. 2) It is capable of performing heterogeneous streaming processing (HSP) in a single hardware, which incorporates streams in different data types with the designed streaming interface. This could further reduce the bandwidth requirement, reduce the power consumption, and increase the throughput. 3) It employs sub-word level parallelism (SLP) to reduce the bandwidth requirement, reduce power consumption, increase the processing throughput, and improve the hardware and memory sharing. 
This paper is organized as follows. In Section II, the proposed CRISPA is described and compared with conventional SIMD array. The design techniques of HSP and SLP are then introduced, and the overall architectures of the proposed processor are also introduced in this section. Section III presents one design example with the proposed processor. Next, Section IV shows the implementation results and the comparisons with the state-of-the-art vision processors and media processors. Finally, the paper is concluded in Section V.

\section{Proposed HaRdWARE ARChitecture}

\section{A. Proposed Hardware Architecture}

The concept of coarse-grained reconfigurable image stream processing architecture (CRISPA) [13] is shown along with the conventional single-instruction-multiple-data (SIMD) array in Fig. 2. In the vision processors with SIMD array [9] [10] [12], for increasing the hardware utilization, very large on-chip memories with high off-chip bandwidth are utilized, which usually leads to high power and cost as shown in Fig. 2(a). In contrast, the CRISPA is mainly consist of two parts: one is the Reconfigurable Stream Processing Elements (RSPE) and the other is the Reconfigurable Interconnection (RI). The RSPEs are the reconfigurable fabrics that can process data streams with user-specified operations. The RI is employed to transport the data streams among RSPEs or I/O interfaces. After the configuration is loaded, CRISPA process the image data stream in a pipelined manner as shown in Fig. 2(b), which makes the large on-chip memories with high off-chip bandwidth no longer required since the effective memory bandwidth is increased multiple times by passing the image stream between RSPEs in the pipeline. The cost and power consumption can be thus largely reduced when compared to the conventional SIMD array.

In Fig. 3, based on CRISPA, the architecture of the proposed reconfigurable stream processor for distributed smart cameras is shown. It serves as a co-processor in the systemon-a-chip (SoC) of a smart camera. The users can program the reconfigurable stream processor via writing the configurations into the Context Registers (CR). The Main Controller reads the configurations in the $\mathrm{CR}$ to control the whole system, including the configurations of the RI and the configurations of the datapath in each RSPE. The control signals are indicated with the dashed lines in Fig. 3. The RI is composed of hierarchies of multiplexers, and the input selection for these multiplexers are controlled by the configurations in the CR. A hierarchical model of the RI is shown in Fig. 4. In Fig. 4, the input data of a Destination RSPE is selected from the outputs of many Source RSPEs by the signal Select RSPE, and the signal Select Output is designed to fulfill the required precision of the Destination RSPE by selecting required data in bit-level from each Source RSPE. After the RI is configured, the data can be streamed from one RSPE to another in a pipelined manner as shown in Fig. 2(b).

To efficiently address the heterogeneous data types in smart camera applications, in addition to CRISPA, Sub-word Level Parallelism (SLP) and Heterogeneous Streaming Processing (HSP) architectures are also proposed. Figure 5 shows the design concepts of SLP and and the proposed streaming interface (SI) for HSP.
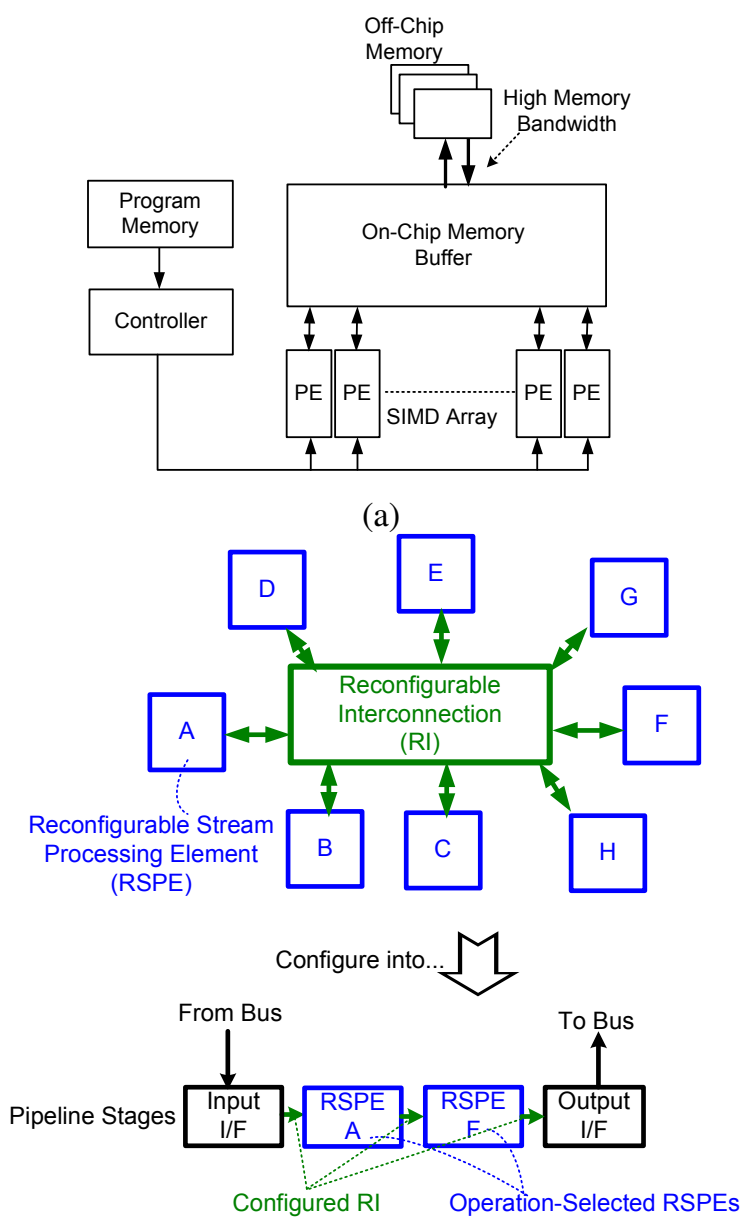

(b)

Fig. 2. Architectural concept of (a) conventional SIMD array and (b) proposed CRISPA.

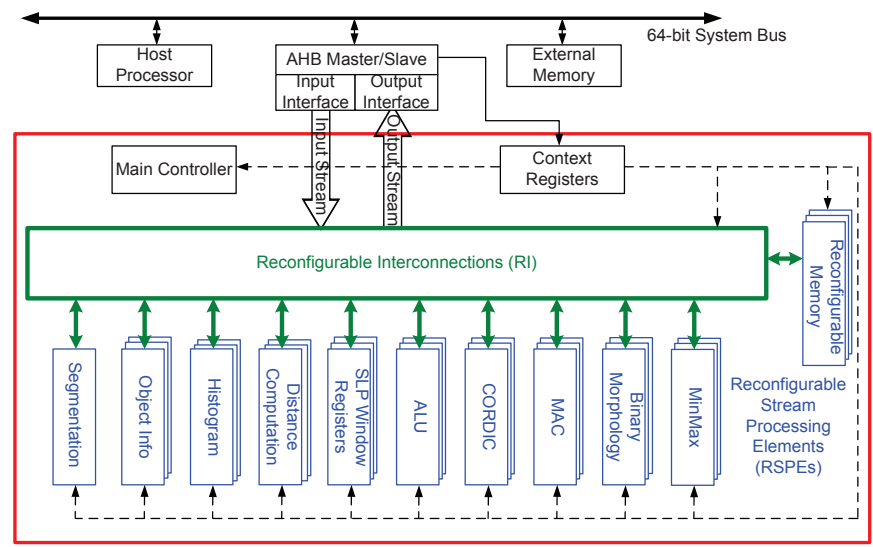

Fig. 3. Block diagram of the proposed coarse-grained reconfigurable stream processor for smart cameras.

SLP architecture is shown in Fig. 5(a). The basic concept is to change the parallelism of function units (FUs) inside RSPEs to fit the bus bit-width of data stream. With SLP Data Access and SLP Data Processing, the hardware can access and process more than one data simultaneously. As a result, the bus utilization and throughput are increased. For example, in Fig. 


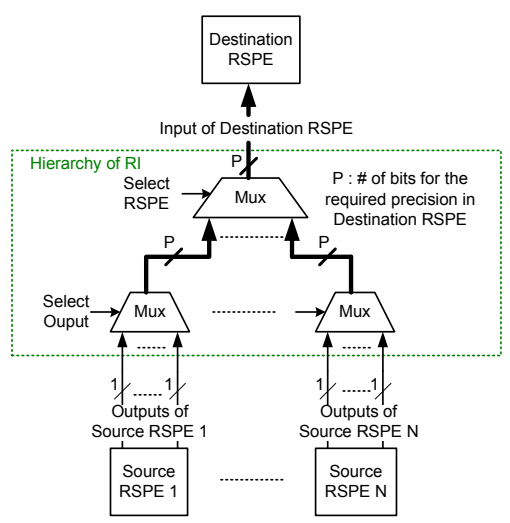

Fig. 4. Hierarchical Model of the RI.

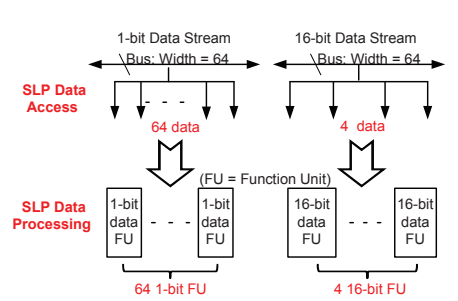

(a)

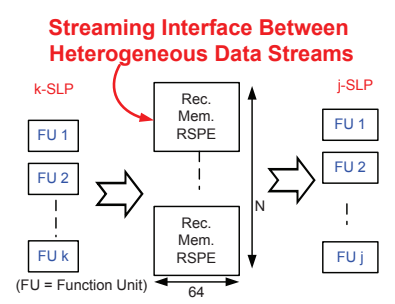

(b)
Fig. 5. (a) SLP. (b) Streaming interface (SI) for HSP.

5(a), up to 64 1-bit data or four 16-bit data can be accessed and processed simultaneously in the case of 64-bit bus. The processing throughput and the bus utilization are increased by 64 and 4 times, respectively.

In Fig. 5(b), the SI for HSP is designed by dynamically allocating buffers with Reconfigurable Memory (RM) RSPE when the levels of SLP parallelism between two pipeline stages, are not equal (e.g. $j \neq k$ in Fig. 5(b)). With SI, streams with different data types can be converted on-chip without the need to write the intermediate data to the off-chip memory and read it back. Therefore, the off-chip memory bandwidth, on-chip memory size, and power consumption can be reduced.

\section{B. RSPES}

After analyzing the operations in computer vision algorithms for a smart camera, we design 11 different types of RSPEs for different types of operations and data access patterns, as shown in Table I. Note that, the numbers of RSPEs can be decided according to the target specification of the considered smart camera applications. If higher specifications are required, more RSPEs can be added. In these RSPEs, some of them are designed with SLP inside RSPE. Moreover, with the proposed CRISPA, multiple RSPEs can be employed simultaneous in a pipelined/parallel manner with the configuration of RI to provide parallelism. Furthermore, through the configuration of RI, the data with different word-length can be fed into RSPEs, making it an SLP reconfigurable device with much better power efficiency and area efficiency than processor based approaches.

For the 11 RSPEs, short descriptions of their functions are provided in Table I. More architecture details of the Reconfig-
TABLE I. SUMMARY OF RSPES

\begin{tabular}{|l|l|}
\hline \multicolumn{1}{|c|}{ RSPE } & \\
\hline Reconfigurable Memory & For any memory usage in ReSSP and for any data type (for any-SLP) \\
\hline MinMax & 27 inputs, find the maximum and minimum (1-SLP) \\
\hline Binary Morphology & Dilation, erosion, conditional erosion with 64 operations in parallel (64-SLP) \\
\hline MAC & support multiply-and-accumulate operations(1-SLP) \\
\hline CORDIC & Using CORDIC arithmetic to support trigonometric, exponential, ... etc (1-SLP) \\
\hline ALU & Arithmetic and logical operations (1-SLP) \\
\hline SLP Window Registers & Data ordering for SLP window operations (for any-SLP) \\
\hline Distance Computation & Calculate the distances(dissimilarity) between two distributions(histograms) (2-SLP) \\
\hline Histogram & Distribution (histogram) accumulation, such as color, gradient ...etc (2-SLP) \\
\hline Object Info & Support object level information extraction with 8 pixels processed in parallel (8-SLP) \\
\hline Segmentation & Video object segmentation, feedbacks from high level algorithms are supported(1-SLP) \\
\hline
\end{tabular}

urable Memory RSPE, MAC RSPE, and Binary Morphology RSPE are shown below.

Figure 6(a) shows the detailed architecture of the Reconfigurable Memory (RM) RSPE. The RM RSPE is an on-chip memory. The word-length of RM RSPE is 64-bit and its word number is 10 . Note that the word-length is equal to the width of the system bus for memory sharing with any data types and for the convenience of data transaction through bus. For this, multiple data can be accessed through the bus at one cycle and stored in the same word of RM RSPE, which is called SLP data access. The reconfigurability of RM RSPE comes from the configuration of the RI. When larger word number is required, multiple RM RSPEs can be connected with the RI. For example, in Fig. 6(a), one Reconfigurable Memory RSPE can be used as the delay line for a 640x480 binary image, while the delay line for a $640 \times 480$ gray-scale image is formed with 8 Reconfigurable Memory RSPEs. Note that it employs 8-level SLP data transfer in the delay line for gray scale image, since the data size is 8-bit. The RM RSPEs can be used for any purposes, any data types and any level of SLP, playing the roles of I/O buffer, SI, delay lines, and storages for histograms, while the single RM RSPE serves as the basic building block for these memory functions. We call this powerful memory sharing design as SLP memory sharing, since the SLP data access, the SLP on-chip storage, and the SLP data processing can be realized by building target memory functions with the RM RSPEs as building blocks through the configurability of RI.

Figure 6(b) shows the MAC RSPE, which is used to support Multiply-and-Accumulate-Based (MAC-Based) kernel operations. There are nine multipliers in order to efficiently support image filtering operations in $3 \times 3$ windows. Multiple MAC RSPEs can be employed together for larger window sizes. The filter coefficients are stored in the CR, while the MAC RSPE reads them through input selection provided by RI. The data to be processed are input from RM RSPEs or other RSPEs, and the selection is done by RI and controlled by CR.

Figure 7 shows the architecture of the Binary Morphology RSPE. It is used to accelerate the binary morphology operations widely used in many computer vision algorithms, and is designed with 64-level SLP. The shaded area is for boundary condition handling, where $\mathrm{VCx}$ signals are for vertical control and $\mathrm{HCx}$ signals are for horizontal control. Dilation/Erosion, Conditional Erosion Enable, and Morphological Operation/No 

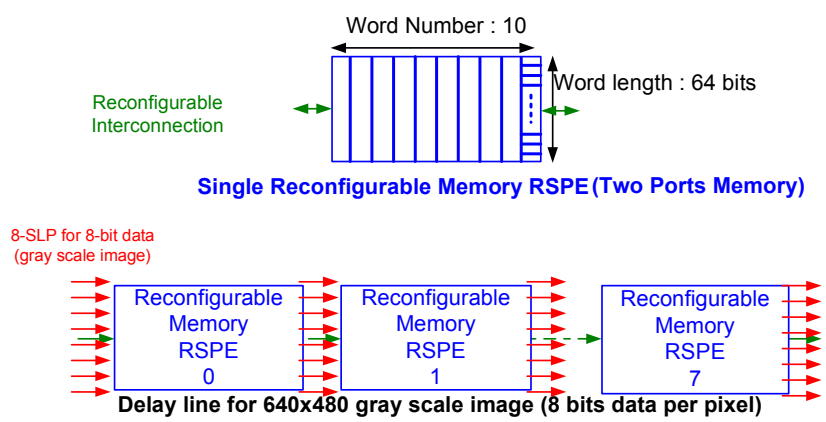

(a)

From Context Registers, Reconfigurable Memory RSPE, or Other RSPEs

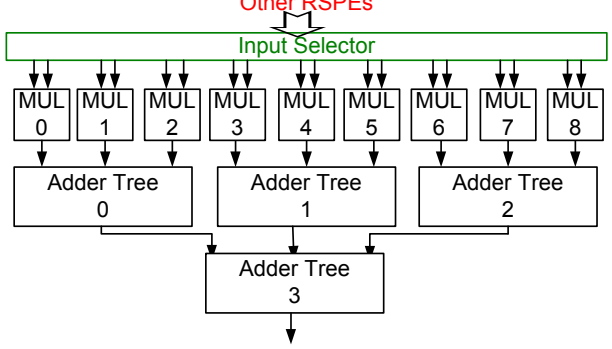

(b)

Fig. 6. (a) Reconfigurable Memory RSPE and (b) MAC RSPE.

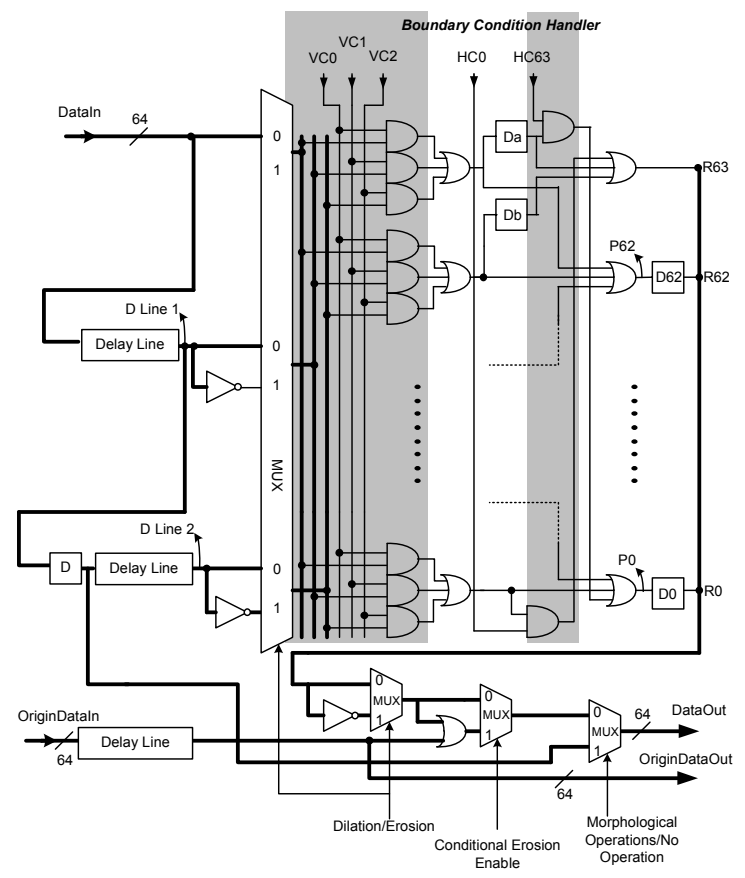

Fig. 7. Detailed architecture of the Binary Morphology RSPE.

Operation are the control signals to program this RSPE to achieve one of the four operations: $3 \times 3$ Dilation, $3 \times 3$ Erosion, $3 \times 3$ Conditional Erosion (Geodesic Erosion), and No Operation (NOP). The control signals are stored in the Context Register. With the SLP architecture, this RSPE can process $3 \times 3$ binary morphological operation for 64 pixels at the same time. Since almost all kinds of morphological operations can be decomposed into simple dilation and erosion operations, by connecting such RSPEs in pipeline, it can accelerate many applications with morphological operations. Note that for some complex morphological operations, when the number of required RSPEs exceeds that in a fabricated chip, this RSPE array can be executed iteratively with folding technique via the off-chip frame buffer, which is the temporal resource sharing ability introduced by the reconfigurable architecture.

\section{EXAMPLE SMART CAMERA APPLICATION}

One example application for the proposed reconfigurable stream processor is illustrated in Fig. 8. Note that, since the proposed reconfigurable stream processor plays the role of a co-processor, it only takes charge of the computationally intensive operations for real-time acceleration, and the other operations can still be executed with the Host Processor (ARM926EJ-S). In order to minimize the necessary data transaction with external memory through the system bus to reduce the bus bandwidth consumption and processing time, when designing the configurations for the reconfigurable stream processor, we tend to execute as many operations (or as many functions) as possible in a single configuration setting before outputting the result or intermediate result to the offchip memory. Here, we call the processing under a single configuration setting as a "Time Frame."

In Fig. 8, it shows the Algorithm Flowchart, Result After Time Frame, and Hardware Interconnection Configuration of the target application: Video Object Segmentation and Tracking [14] [15] [16]. In Time Frame 1 (Segmentation), the Segmentation RSPE is used to support background subtraction video object segmentation, and the object mask of the foreground moving object is generated. The input data (current frame and background model) and output result (object mask and updated background model) are stored temporarily in RM RSPEs for I/O buffering. In Time Frame 2 (Morphology Denoise), the RM RSPEs and Window Register RSPEs are used to provide $3 \times 3$-window data organization for the object mask. Then the Binary Morphology RSPEs are used to denoise the object mask. In Time Frame 3 (Connected Component Labeling), each of the foreground objects on the de-noised object mask is given an unique label with connected component labeling algorithm, where the ALU RSPEs are employed. Note that, since the input of this time frame is 1-bit binary object mask with 64 data input/processed at the same time while the label number is 8 -bit per data, where only 8 data can be transferred through 64-bit bus at a time, a streaming interface should be applied here. The RM RSPEs play the role of streaming interface and output buffering at the same time. In Time Frame 4 (Tracking Initialization), an image plane labeled as objects (result of Time Frame 3 ) is input to the Object Info RSPE for extracting the object location and bounding box. After comparing the object locations in previous frames and the currently extracted object information, new trackers could be initialized on locations of the new objects (not appear at previous frames). In Time Frame 5 (Particle Filter), we support the computation-intensive importance measurement step in particle filter. The importance measurement step in particle filter matches the image patch of a particle with object model in order to find out the weight of each particle, and this critical step decides the performance of a particle filter based tracker. Here, the matching is performed by comparing the color histograms of a particle's image patch and the object 


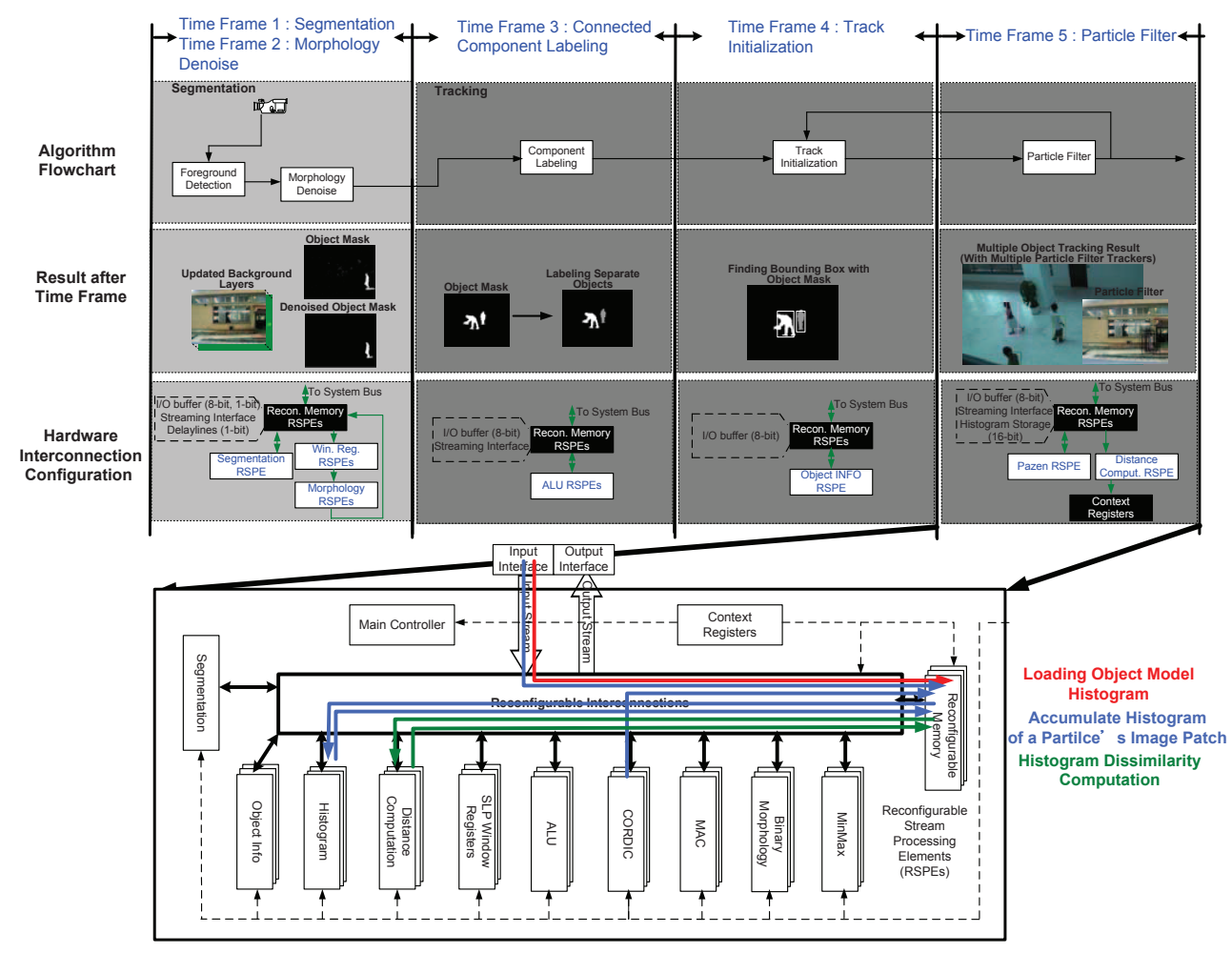

Fig. 8. Example application of the proposed reconfigurable stream processor: Video object segmentation and tracking.

model. At the bottom of Fig. 8, the related data flows are also shown.

In Fig. 8, we can see that the hardware interconnection configurations are different in different time frames, in which the RSPEs and the interconnections have different configurations to process different tasks. We can also see that the Reconfigurable Memory RSPEs are used in processing tasks with different data sizes, playing the roles of I/O buffer, Streaming Interfaces, delay lines, and storages for histogram.

\section{IMPLEMENTATION RESULTS AND COMPARISONS}

A prototype chip of the proposed design is fabricated with TSMC 90nm technology. The chip specification is shown at the right-most column of Table II. The chip area is $10.4 \mathrm{~mm}^{2}$ with $0.9 \mathrm{M}$ gates, and the maximum working frequency is $149 \mathrm{MHz}$. The chip micrograph is shown in Fig. 9.

In Table III, it shows that for the Video Object Segmentation, the proposed reconfigurable processor provides a throughput of 640x480 125fps. While running both Video Object Segmentation and Video Object Tracking (Particle Filter), the processing throughput is $640 \times 480$ 30fps and 11 objects can be tracked at the same time, where 33,000 particles are processed per second. For face detection, scoring and ranking [17], it achieve 150 faces per second. Finally, for the SIFT [18], it can support $1920 \times 1080$ full-HD object recognition in realtime. For low-level operations, the proposed processor can process $4431920 \times 1080$ images per second for $7 \times 7$ Gaussian Filter. For morphological operation, with 16 64-SLP Binary Morphology RSPEs, 56,722 full-HD $1920 \times 1080$ frames can be processed per second. For Histogram Accumulation, the histograms of $37,48880 \times 80$ image blocks can be provided per second by processing two pixels simultaneously. Moreover, three histograms can be accumulated at the same time.

Table II shows the chip specifications of this work and the previous works [10] [11] [12] for comparison. It can be observed that the peak performance of the proposed reconfigurable stream processor is higher than the state-of-the-art chips. In most vision processors, the on-chip memories are very large to fulfill the high parallelism. As shown in Table II, with SLP memory sharing, the on-chip memory usage is 18.2 to 87.4 times smaller than the state-of-the-art chips. With the much more efficient memory scheme, CRISPA, and speciallydesign RSPEs, the area efficiency is 3.8 to 25.5 times higher than other chips. The low power consumption of our design is achieved by using the CRISPA with optimized RSPEs. Moreover, clock gating is also employed for unused RSPEs in each time frame. The power efficiency is 4.5 to 12.5 times better than the state-of-the-art chips. Note that the comparison may be not fully fair because the function of these chips are different; however, the efficiency of the proposed architecture can still be revealed in this comparison.

\section{CONClusion}

In this work, an efficient reconfigurable stream processor for distributed smart cameras is proposed. It is designed with the proposed coarse-grained reconfigurable image stream processor architecture (CRISPA), heterogeneous streaming processing (HSP) and sub-word level parallelism (SLP) architectures. With these design techniques, the proposed processor outperforms the vision processors designed with conventional SIMD array hardware architecture in terms of throughput, 
TABLE II. CHIP SPECIFICATIONS AND COMPARISONS

\begin{tabular}{|c|c|c|c|c|}
\hline Work & {$[10]$} & [11] & {$[12]$} & Proposed \\
\hline Process & $\begin{array}{l}\text { UMC 0.18um } \\
\text { 2P4M CMOS } \\
\text { Image Sensor } \\
\text { Process }\end{array}$ & $\begin{array}{l}\text { TSMC 90nm } \\
\text { IP9M CMOS }\end{array}$ & $\begin{array}{l}0.13 \mu \mathrm{m} 8 \text { metal } \\
\text { CMOS }\end{array}$ & $\begin{array}{l}\text { TSMC 90nm 1P9M } \\
\text { CMOS }\end{array}$ \\
\hline Die Size & $\begin{array}{c}70.5 \mathrm{~mm}^{2} \\
\text { (core area) }\end{array}$ & $28 \mathrm{~mm}^{2}$ (die size) & $50 \mathrm{~mm}^{2}$ (die size) & $\begin{array}{c}10.4 \mathrm{~mm}^{2} \\
(3.2 \mathrm{~mm} \times 3.2 \mathrm{~mm})\end{array}$ \\
\hline Power Supply & $2.1 \mathrm{~V}$ & $\begin{array}{c}\text { Core } 1.2 \mathrm{~V}, \mathrm{I} / \mathrm{O} \\
2.5 \mathrm{~V}\end{array}$ & $0.65 \mathrm{~V}-1.2 \mathrm{~V}$ & Core $1.2 \mathrm{~V}, \mathrm{l} / \mathrm{O} 2.5 \mathrm{~V}$ \\
\hline Total Gate Count & N/A & $3.00 \mathrm{M}$ gates & $2.92 \mathrm{M}$ gates & $\begin{array}{l}\text { 0.9M Gates (2-Input } \\
\text { NAND Gate, Including } \\
\text { On-Chip Memory) }\end{array}$ \\
\hline On-Chip Memory (Kb) & 1,024 & 1,192 & 4,896 & $\begin{array}{c}56 \text { (Including Context } \\
\text { Registers) }\end{array}$ \\
\hline Working Frequency & $50 \mathrm{MHz}$ & $\mathrm{Max} 200 \mathrm{MHz}$ & $\begin{array}{c}\text { NoC: } 400 \mathrm{MHz} \\
\text { Processing: Max } \\
\text { 200MHz }\end{array}$ & $\mathrm{Max} 149 \mathrm{MHz}$ \\
\hline $\begin{array}{l}\text { Peak Performance } \\
\text { (GOPS) }\end{array}$ & 76.8 & 814 & 228 & 1157.82 \\
\hline Power Consumption & $\begin{array}{c}\text { Vision Processor: } \\
374 \mathrm{~mW}\end{array}$ & $1214 \mathrm{~mW}$ (Peak) & $\begin{array}{c}\text { Peak : } 704 \mathrm{~mW} \\
\text { Average: } 345 \mathrm{~mW}\end{array}$ & $197 \mathrm{~mW}$ (peak) \\
\hline $\begin{array}{l}\text { Area Efficiency } \\
\left(\text { GOPS } / \mathrm{mm}^{\wedge} 2\right)\end{array}$ & 4.357 & 29.100 & 9.120 & 111.329 \\
\hline $\begin{array}{l}\text { Power Efficiency } \\
\text { (TOPS/W) }\end{array}$ & 1.283 & 0.671 & 0.469 & 5.877 \\
\hline $\begin{array}{l}\text { Resolution and Spec } \\
\text { for Image Analysis }\end{array}$ & $\begin{array}{c}\text { Target at } 128 \times 128 \\
\text { image analysis }\end{array}$ & \begin{tabular}{|c|} 
Target at \\
$160 \times 120$ \\
Image analysis
\end{tabular} & $\begin{array}{c}\text { SIFT } 640 \times 480 \\
30 \text { fps and object } \\
\text { recognition }\end{array}$ & $\begin{array}{l}\text { SIFT } 640 \times 48030 \mathrm{fps} \\
\text { and other applications } \\
\text { with high spec }\end{array}$ \\
\hline
\end{tabular}

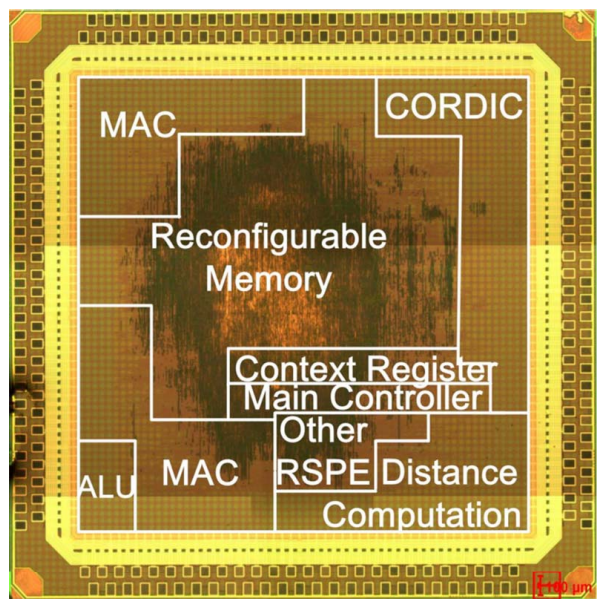

Fig. 9. Die photo.

power efficiency and area efficiency. In the implementation results, this work achieves high-throughput image analysis with much lower on-chip memory usage (18.2 to 87.4 times), much higher area efficiency (3.8 to 25.5 times), and much higher power efficiency (4.5 to 12.5 times) than the state-ofthe-art chips.

\section{ACKNOWLEDGMENT}

The authors would like to thank TSMC University Shuttle Program and Morly Hsieh for process support and also thank

TABle III. Performance of the Proposed Processor.

\begin{tabular}{|c|c|c|}
\hline $\begin{array}{l}\text { Smart Camera } \\
\text { Application }\end{array}$ & $\begin{array}{l}\text { Supported High } \\
\text { Level Algorithm }\end{array}$ & Specification \\
\hline $\begin{array}{l}\text { Video Object Segmentation } \\
\text { and Tracking }\end{array}$ & $\begin{array}{l}\text { Segmentation : Multi-Layer } \\
\text { Background Subtraction } \\
\text { Tracking : Particle Filter }\end{array}$ & $\begin{array}{l}\text { Segmentation : } 640 \times 480125 \mathrm{fps} \\
\text { Segmentation + tracking : } 640 \times 48030 \mathrm{fps} 11 \text { Objects } \\
\text { (or } 33000 \text { particles per sec) with object size } 80 \times 80\end{array}$ \\
\hline $\begin{array}{l}\text { Face Detection, Scoring and } \\
\text { Ranking }\end{array}$ & $\begin{array}{l}\text { Face Detection with } \\
\text { Segmentation and } \\
\text { Feature-based Face Scoring }\end{array}$ & 150 faces per second \\
\hline $\begin{array}{l}\text { Object Detection and } \\
\text { Recognition }\end{array}$ & $\begin{array}{l}\text { Scale Invariant Feature } \\
\text { Transform (SIFT) }\end{array}$ & $\begin{array}{l}\text { can support } 1920 \times 1080 \text { full HD object recognition in } \\
\text { real-time }\end{array}$ \\
\hline
\end{tabular}

Chip Implementation Center (CIC) for design flows and chip testing. This work is funded by National Science Council and TSMC.

\section{REFERENCES}

[1] ITU Internet reports 2005: The Internet of Things. [Online]. Available: http://www.itu.int/internetofthings/

[2] G. Lawton, "Machine-to-machine technology gears up for growth," IEEE Computer, vol. 37, no. 9, pp. 12-15, Sep. 2004.

[3] B. Rinner and W. Wolf, "An introduction to distributed smart cameras," Proc. IEEE, vol. 96, no. 10, pp. 1565 - 1575, Oct. 2008.

[4] W. Wolf, B. Ozer, and T. Lv, "Smart cameras as embedded systems," IEEE Computer, vol. 35, no. 9, pp. 48-53, Sep. 2002.

[5] G. Foresti, C. Micheloni, L. Snidaro, P. Remagnino, and T. Ellis, "Active video-based surveillance systems: the low-level image and video processing techniques needed for implementation," IEEE Signal Processing Mag., vol. 22, no. 2, pp. 25-37, Mar. 2005.

[6] W.-K. Chan, J.-Y. Chang, T.-W. Chen, Y.-H. Tseng, and S.-Y. Chien, "Efficient content analysis engine for visual surveillance network," IEEE Trans. Circuits Syst. Video Technol., vol. 19, no. 5, pp. 693-703, May 2009.

[7] S.-Y. Chien and W.-K. Chan, Video Surveillance. Chapter 7 : Cooperative Visual Surveillance Network with Embedded Content Analysis Engine. InTech, 2011.

[8] S.-Y. Chien, T.-Y. Cheng, S.-H. Ou, C.-C. Chiu, C.-H. Lee, V. Somayazulu, and Y.-K. Chen, "Power consumption analysis for distributed video sensors in machine-to-machine networks," IEEE J. Emerging and Selected Topics in Circuits and Systems, vol. 3, no. 1, pp. 55-64, Mar. 2013.

[9] A. Abbo and et al., "Xetal-II: A 107GOPS, 600mW massively-parallel processor for video scene analysis," in Dig. Tech. Papers IEEE International Solid-State Circuits Conference, 2007, pp. 270-271.

[10] C.-C. Cheng and et al., "iVisual: An intelligent visual sensor SoC with 2790fps CMOS image sensor and 205GOPS/W vision processor," in Dig. Tech. Papers IEEE International Solid-State Circuits Conference, 2008, pp. $306-615$.

[11] T.-W. Chen and et al., "A multimedia semantic analysis SoC (SASoC) with machine-learning engine," in Dig. Tech. Papers IEEE International Solid-State Circuits Conference, 2010, pp. 338 - 339.

[12] S. Lee and et al., "A $345 \mathrm{~mW}$ heterogeneous many-core processor with an intelligent inference engine for robust object recognition," in Dig. Tech. Papers IEEE International Solid-State Circuits Conference, 2010, pp. $332-333$.

[13] J. C. Chen and S.-Y. Chien, "CRISP: Coarse-grained reconfigurable image stream processor for digital still cameras and camcorders," IEEE Trans. Circuits Syst. Video Technol., vol. 18, no. 9, pp. 1223-1236, Sep. 2008.

[14] W.-K. Chan and S.-Y. Chien, "Real-time memory-efficient video object segmentation in dynamic background with multi-background registration technique," in Proc. IEEE Multimedia Signal Processing Workshop, Oct. 2007.

[15] K. Nummiaro, E. Koller-Meier, and L. V. Gool, "An adaptive colorbased particle filter," Image and Vision Computing, vol. 21, no. 1, pp. 99-110, Jan. 2003.

[16] E. Maggio, F. Smerladi, and A. Cavallaro, "Adaptive multifeature tracking in a particle filtering framework," IEEE Trans. Circuits Syst. Video Technol., vol. 17, no. 10, pp. 1348-1359, Oct. 2007.

[17] T.-W. Chen, W.-K. Chan, and S.-Y. Chien, "Efficient face detection with segmentation and feature-based face scoring in surveillance systems," in Proc. IEEE Multimedia Signal Processing Workshop, Oct. 2007.

[18] D. G. Lowe, "Distinctive image features form scale-invariant keypoints," Int. J. of Computer Vision, 2004. 